\title{
Design Criteria for Star Polymer Formation Processes via Living Free Radical Polymerization (RAFT)
}

\author{
Hugh Chaffey-Millar ${ }^{\mathrm{a}}$, Martina Stenzel ${ }^{\mathrm{a}}$, Thomas P. Davis ${ }^{\mathrm{a}}$, \\ Michelle L. Coote ${ }^{\mathrm{b}}$, Christopher Barner-Kowollik*a
}

${ }^{a}$ Centre for Advanced Macromolecular Design, School of Chemical Sciences and Engineering, The University of New South Wales, Sydney, NSW 2052, Australia, camd@unsw.edu.au

${ }^{\mathrm{b}}$ ARC Centre of Excellence in Free-Radical Chemistry and Biotechnology, Research School of Chemistry, Australian National University, Canberra ACT 0200, Australia.

SUPPLEMENTARY DATA SECTION 


\title{
S1 B3LYP/6-31G(d) Optimised Geometries
}

See Figure 6 and text of manuscript for further explanation of how these molecular geometries were used.

\section{Open conformation (ROMP2/6-311+G(3df,2p) calculation)}

\begin{abstract}
$1 \backslash 1 \backslash G I N C-A C 45 \backslash S P \backslash R O M P 2-F C \backslash 6-311+G(3 d f, 2 p) \backslash C 15 H 13 S 2$ (2) \HWC561 $27-A u g-20$ $05 \backslash 0 \backslash \backslash \#$ ROMP 2/6-311+G (3DF, 2P) MAXDISK=2684354560 MAXDISK=2684354560 MA $\mathrm{XDISK}=2684354560 \backslash \backslash$ open star precursor $\backslash \backslash 0,2 \backslash \mathrm{C}, 0,-2.724237799,-0.3449186$ $787,-0.0909786251 \backslash \mathrm{C}, 0,-3.3004117489,0.570138329,0.8585077287 \backslash \mathrm{C}, 0,-4.57$ $89235606,1.1248661794,0.5453889055 \backslash \mathrm{C}, 0,-5.2425619931,0.813024711,-0.62$ $78317331 \backslash \mathrm{C}, 0,-4.6622858269,-0.072277525,-1.5470499623 \backslash \mathrm{C}, 0,-3.413897092$ $5,-0.6368938624,-1.2654520669 \backslash \mathrm{C}, 0,-2.6636187864,0.9290865699,2.0588416$ $569 \backslash \mathrm{H}, 0,-5.0283587552,1.8111075555,1.2586414107 \backslash \mathrm{H}, 0,-6.2135216178,1.25$ $47244073,-0.8351369963 \backslash \mathrm{H}, 0,-5.1760778634,-0.3230263325,-2.47037062 \backslash \mathrm{H}, 0$ $,-2.9676199023,-1.3278839381,-1.9769431933 \backslash \mathrm{C}, 0,-1.3945291818,-1.004360$ $4026,0.1586269173 \backslash \mathrm{S}, 0,-0.0197669899,0.1543699654,-0.2723108213 \backslash \mathrm{C}, 0,1.4$ $341559202,-0.7568252997,0.1428734347 \backslash \mathrm{S}, 0,1.4306680354,-2.332423155,0.6$ $528037093 \backslash \mathrm{C}, 0,2.6794870582,0.0357475324,-0.0392971899 \backslash \mathrm{C}, 0,2.7125901344$ $, 1.425886355,0.181009476 \backslash \mathrm{C}, 0,3.8954551483,2.1427518229,0.0108652422 \backslash \mathrm{C}$, $0,5.0610123463,1.4896274148,-0.3920680113 \backslash \mathrm{C}, 0,5.0398761978,0.110241411$ $9,-0.6158736883 \backslash \mathrm{C}, 0,3.8645832147,-0.6114797199,-0.4353799037 \backslash \mathrm{H}, 0,1.819$ $1530109,1.9413170295,0.5184388305 \backslash \mathrm{H}, 0,3.9063258698,3.2124224118,0.2009$ $28158 \backslash \mathrm{H}, 0,5.9816231925,2.0505080995,-0.5282285742 \backslash \mathrm{H}, 0,5.9425468134,-0$. $4047982056,-0.9326428044 \backslash \mathrm{H}, 0,3.8433043471,-1.6824395562,-0.605377248 \backslash \mathrm{H}$ $, 0,-3.1416876518,1.6208101394,2.7447275927 \backslash \mathrm{H}, 0,-1.6877510154,0.5537458$ $511,2.3400658085 \backslash \mathrm{H}, 0,-1.2530100754,-1.3081479934,1.1991943921 \backslash \mathrm{H}, 0,-1.2$ $703833192,-1.8957279215,-0.460239199 \backslash \backslash$ Version=IA64L-G03RevC.02 $\backslash$ State $=2$ $-\mathrm{A} \backslash \mathrm{HF}=-1370.797404 \backslash \mathrm{MP} 2=-1373.5114942 \backslash \mathrm{RMSD}=7.561 \mathrm{e}-09 \backslash \mathrm{PG}=\mathrm{C} 01 \quad[\mathrm{X}(\mathrm{C} 15 \mathrm{H} 13 \mathrm{~S} 2$ )$] \backslash \backslash @$
\end{abstract}

\section{Closed conformation (ROMP2/6-311+G(3df,2p) calculation)}

$1 \backslash 1 \backslash G I N C-A C 45 \backslash S P \backslash R O M P 2-F C \backslash 6-311+G(3 d f, 2 p) \backslash C 15 H 13 S 2$ (2) HWC 561 $\backslash 27-A u g-20$ $05 \backslash 0 \backslash \backslash \#$ ROMP 2/6-311+G(3DF, 2P) MAXDISK=2684354560\\closed star precurso $r \backslash \backslash 0,2 \backslash \mathrm{C}, 0,3.2287537768,1.2105022807,0.0956632541 \backslash \mathrm{C}, 0,2.4854405867,0.0$ $008788462,-0.0000419305 \backslash \mathrm{C}, 0,3.2297360168,-1.2081466126,-0.095728621 \backslash \mathrm{C}$, $0,4.6178783261,-1.1998563292,-0.0983854925 \backslash \mathrm{C}, 0,5.3276676942,0.00202923$ $42,-0.0002796096 \backslash \mathrm{C}, 0,4.616919374,1.203329036,0.0979537815 \backslash \mathrm{C}, 0,1.045885$ $9333,0.0002894515,-0.0000431648 \backslash S, 0,0.1389867409,-1.4127906899,-0.5426$ $103218 \backslash \mathrm{C}, 0,-1.284141754,-1.4273600418,0.6366453158 \backslash \mathrm{C}, 0,-2.5329123346,-$ $0.6618980236,0.2477168678 \backslash \mathrm{C}, 0,-3.7618945983,-1.2974788787,0.4715465828$ $\backslash \mathrm{C}, 0,-4.9772075749,-0.6562138884,0.2372784793 \backslash \mathrm{C}, 0,-4.9775820209,0.6538$ $100666,-0.2344928816 \backslash \mathrm{C}, 0,-3.7626349597,1.2952182165,-0.4702349291 \backslash \mathrm{C}, 0$, $-2.5333140412,0.6598335268,-0.2478042809 \backslash C, 0,-1.2849986312,1.425366757$ 
$4,-0.637845601 \backslash \mathrm{S}, 0,0.1375906332,1.4127328131,0.5418512556 \backslash \mathrm{H}, 0,-5.91239$ $98186,1.1755158,-0.4197984676 \backslash \mathrm{H}, 0,-5.9117338891,-1.1779930737,0.423850$ $1 \backslash \mathrm{H}, 0,-3.7625312563,-2.3183165007,0.8471639862 \backslash \mathrm{H}, 0,-3.7637924649,2.316$ $1182839,-0.8456757594 \backslash \mathrm{H}, 0,-1.5321109109,2.4847167602,-0.753454381 \backslash \mathrm{H}, 0$, $-0.8915467905,1.0767644923,-1.597184569 \backslash \mathrm{H}, 0,-1.5307073194,-2.487002015$ $3,0.7506768908 \backslash \mathrm{H}, 0,-0.8910201035,-1.0799238135,1.5965607396 \backslash \mathrm{H}, 0,2.6998$ $145302,-2.1530457907,-0.1493660244 \backslash \mathrm{H}, 0,5.1540529368,-2.1429365976,-0.1$ $687988915 \backslash \mathrm{H}, 0,6.4138987789,0.0025113225,-0.0006130551 \backslash \mathrm{H}, 0,5.1523642857$

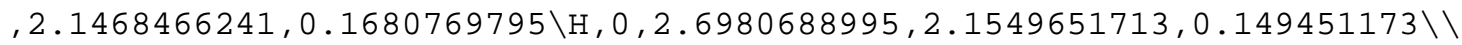
Version=IA 64L-G03RevC.02 State $=2-A \backslash H F=-1370.8079626 \backslash M P 2=-1373.5325635 \backslash$ $\mathrm{RMSD}=3.510 \mathrm{e}-09 \backslash \mathrm{PG}=\mathrm{C} 01 \quad[\mathrm{X}(\mathrm{C} 15 \mathrm{H} 13 \mathrm{~S} 2)] \backslash \backslash \mathrm{e}$

\section{Close-open transition state conformation (ROMP2/6-311+G(3df,2p) calcu- lation)}

$1 \backslash 1 \backslash \mathrm{GINC}-\mathrm{AC} 41 \backslash \mathrm{SP} \backslash \mathrm{ROMP} 2-\mathrm{FC} \backslash 6-311+\mathrm{G}(3 \mathrm{df}, 2 \mathrm{p}) \backslash \mathrm{C} 15 \mathrm{H} 13 \mathrm{~S} 2$ (2) $\backslash$ HWC5 61 $\backslash 28-\mathrm{Apr}-20$ $06 \backslash 0 \backslash \backslash \#$ ROMP $2 / 6-311+G(3 D F, 2 P)$ GEOM=CHECK GUESS=READ MAXDISK=1342177280 $\backslash \backslash G 3(M P 2)-R A D \quad C a l C \backslash \backslash 0,2 \backslash C, 0,2.5984283874,-0.1826159429,-0.7818114648 \backslash \mathrm{C}$ $, 0,2.589380828,-0.1467757553,0.6454727998 \backslash \mathrm{C}, 0,3.8113315748,-0.12136748$ $92,1.3256048214 \backslash \mathrm{C}, 0,5.0345615764,-0.19276248,0.6564182791 \backslash \mathrm{C}, 0,5.048032$ $0795,-0.3155388332,-0.7342534055 \backslash \mathrm{C}, 0,3.8478118484,-0.3171985411,-1.433$ $277151 \backslash \mathrm{C}, 0,1.3535200822,-0.3631744239,1.4959811568 \backslash \mathrm{S}, 0,-0.1606754154,0$ $.6615110495,1.2720549211 \backslash \mathrm{C}, 0,-1.160175629,-0.3022524181,0.1595511821 \backslash \mathrm{S}$ $, 0,-0.5162609369,-1.5550708627,-0.7352539191 \backslash \mathrm{C}, 0,1.4193061587,-0.03464$ $69725,-1.5957511716 \backslash \mathrm{C}, 0,-2.5394185591,0.196187989,0.0044542375 \backslash \mathrm{C}, 0,-3$. $2417887458,-0.0112714096,-1.2019100467 \backslash \mathrm{C}, 0,-4.5388627941,0.4613202723$, $-1.3616543938 \backslash \mathrm{C}, 0,-5.1764945551,1.1458607878,-0.3220961405 \backslash \mathrm{C}, 0,-4.4999$ $794074,1.3517152635,0.8809525143 \backslash \mathrm{C}, 0,-3.1979325937,0.885107433,1.04537$ $01443 \backslash \mathrm{H}, 0,5.9892852713,-0.3967030651,-1.2711419898 \backslash \mathrm{H}, 0,5.9633021749,-0$ $.1756090194,1.2196478412 \backslash \mathrm{H}, 0,3.8058674137,-0.0813429926,2.4127276153 \backslash \mathrm{H}$ $, 0,3.8562828569,-0.3782171016,-2.5187529417 \backslash \mathrm{H}, 0,1.4957743612,-0.274606$ $3757,-2.652836399 \backslash \mathrm{H}, 0,0.6931962149,0.7257442759,-1.3437585803 \backslash \mathrm{H}, 0,1.61$ $09638357,-0.2077918242,2.5476090549 \backslash \mathrm{H}, 0,1.0125523076,-1.3978931198,1.3$ $835954656 \backslash \mathrm{H}, 0,-2.6972024733,1.0287797194,1.9971461009 \backslash \mathrm{H}, 0,-4.989397598$ $2,1.8705690513,1.7006914238 \backslash \mathrm{H}, 0,-6.1921094499,1.5106785567,-0.44869957$ $\backslash \mathrm{H}, 0,-5.0556906692,0.2981724387,-2.3035463071 \backslash \mathrm{H}, 0,-2.7481641145,-0.540$ $3484122,-2.009805915 \backslash \backslash$ Version=IA64L-G03RevC.02 \State $=2-A \backslash H F=-1370.7598$ $501 \backslash \mathrm{MP} 2=-1373.4919362 \backslash \mathrm{RMSD}=5.896 \mathrm{e}-09 \backslash \mathrm{PG}=\mathrm{C} 01 \quad[\mathrm{X}(\mathrm{C} 15 \mathrm{H} 13 \mathrm{~S} 2)] \backslash \backslash \mathrm{Q}$

\section{Open conformation (G3(MP2)-RAD calculation)}

$1 \backslash 1 \backslash$ GINC-LC81 \SP \ROMP 2-FC \GTMP 2 large \C10H11S2 (2) \HWC561 \24-Nov-2005 $\backslash \#$ ROMP2/GTMP2LARGE GEOM=CHECK GUESS=READ MAXDISK=268435456 MAXDISK=26 $8435456 \backslash \backslash \mathrm{G} 3(\mathrm{MP} 2)-\mathrm{RAD} \mathrm{cal} \backslash \mathrm{C} \backslash 0,2 \backslash \mathrm{C}, 0,0.9530004425,0.6148926529,-2.211642$ $2224 \backslash \mathrm{C}, 0,0.9539724881,0.6108127669,-0.8185789555 \backslash \mathrm{C}, 0,2.2078523389,0.60$ $66810916,-0.1127261966 \backslash \mathrm{C}, 0,3.4034832268,0.6266495682,-0.89394297 \backslash \mathrm{C}, 0,3$ $.371360803,0.6355105924,-2.2769634233 \backslash C, 0,2.1411128183,0.6275420364,-2$ 
$.9494479093 \backslash C, 0,-0.3598124861,0.6176129383,-0.0855458588 \backslash S, 0,-0.799840$ $3013,-1.095611502,0.4618731165 \backslash \mathrm{C}, 0,-2.3518684546,-0.8946178371,1.26107$ $2416 \backslash S, 0,-3.1596806234,0.5281061869,1.4520386815 \backslash \mathrm{C}, 0,2.2962266481,0.58$ $54404346,1.2896874306 \backslash \mathrm{C}, 0,-2.8770098752,-2.2202900042,1.7748907018 \backslash \mathrm{H}, 0$ $,-3.8620384195,-2.0823100568,2.2229181943 \backslash \mathrm{H}, 0,-2.1965737192,-2.6356675$ $565,2.5286242184 \backslash \mathrm{H}, 0,-2.9492799316,-2.9526385534,0.9616025209 \backslash \mathrm{H}, 0,-1.1$ $734177679,0.9649311478,-0.7264882882 \backslash \mathrm{H}, 0,-0.351302025,1.2518540485,0.8$ $043883558 \backslash \mathrm{H}, 0,4.3567456806,0.6321263644,-0.3714266395 \backslash \mathrm{H}, 0,4.3002997972$ $, 0.6487860818,-2.840646693 \backslash \mathrm{H}, 0,2.1068016918,0.6348533525,-4.0348518351$ $\backslash \mathrm{H}, 0,-0.0004248488,0.6155975892,-2.7349201697 \backslash \mathrm{H}, 0,1.4251033766,0.55375$ $52654,1.932020869 \backslash \mathrm{H}, 0,3.2665132622,0.5873919188,1.775372624 \backslash \backslash$ Version=I A32L-G03RevC.02 \State=2-A \HF=-1180.2353277\MP $2=-1182.1730722 \backslash \mathrm{RMSD}=2.80$ $7 e-09 \backslash P G=C 01 \quad[X(C 10 H 11 S 2)] \backslash \backslash e$

\section{Closed conformation (G3(MP2)-RAD calculation)}

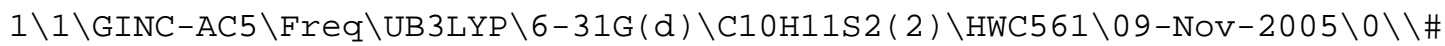
B3LYP / 6-31G* OPT=CALCALL INT (GRID=ULTRAFINE) MAXDISK=134217728 \ $\mathrm{commen}$ t: from nr-star-fused-conf0_chair-calcall-redo2_Cs.zmt \\0,2\H,-3.60481 $98982,0 .,-2.0566436214 \backslash \mathrm{C},-3.6149338907,0 .,-0.9551081416 \backslash \mathrm{C},-2.231550257$ $1,0 .,-0.3553213101 \backslash \mathrm{S},-1.4262702897,-1.5703379517,-0.4109889386 \backslash \mathrm{S},-1.42$ $62702897,1.5703379517,-0.4109889386 \backslash \mathrm{C},-0.1315108093,-1.4835712433,0.91$ $51879134 \backslash \mathrm{C},-0.1315108093,1.4835712433,0.9151879134 \backslash \mathrm{H}, 0.1166808972,-2.5$ $347198217,1.0890640135 \backslash \mathrm{H}, 0.1166808972,2.5347198217,1.0890640135 \backslash \mathrm{H},-0.6$ $186766233,-1.1093197405,1.8207510995 \backslash \mathrm{H},-0.6186766233,1.1093197405,1.82$ $07510995 \backslash C, 1.1072351048,-0.7050646184,0.5600177736 \backslash C, 1.1072351048,0.70$ $50646184,0.5600177736 \backslash \mathrm{C}, 2.2808653835,-1.3861330765,0.2177685013 \backslash \mathrm{C}, 2.28$ $08653835,1.3861330765,0.2177685013 \backslash \mathrm{H}, 2.2798899653,-2.4737625835,0.2172$ $256455 \backslash \mathrm{H}, 2.2798899653,2.4737625835,0.2172256455 \backslash \mathrm{C}, 3.4448022022,-0.6970$ $407465,-0.1221114783 \backslash \mathrm{C}, 3.4448022022,0.6970407465,-0.1221114783 \backslash \mathrm{H}, 4.344$ $6816479,-1.2471770967,-0.3830614805 \backslash \mathrm{H}, 4.3446816479,1.2471770967,-0.383$ $0614805 \backslash \mathrm{H},-4.1687401465,-0.8891940038,-0.6337223541 \backslash \mathrm{H},-4.1687401465,0$. $8891940038,-0.6337223541 \backslash \backslash$ Version=IA64L-G03RevC.02 $\backslash$ State $=2-A^{\prime} \backslash H F=-1184$ $.0242456 \backslash \mathrm{S} 2=0.75529 \backslash \mathrm{S} 2-1=0 . \backslash \mathrm{S} 2 \mathrm{~A}=0.750021 \backslash \mathrm{RMSD}=3.794 \mathrm{e}-09 \backslash \mathrm{PG}=\mathrm{CS} \quad[\mathrm{SG}(\mathrm{C} 2 \mathrm{H} 1$ )$, \mathrm{X}(\mathrm{C} 8 \mathrm{H} 10 \mathrm{~S} 2)] \backslash \backslash \mathrm{Q}$

\section{- $\mathrm{CH}_{3}$ (W1 calculation)}

$1 \backslash 1 \backslash G I N C-S C 64 \backslash F O p t \backslash U B 3 L Y P \backslash 6-31 G(d) \backslash C 1 H 3(2) \backslash H W C 561 \backslash 01-F e b-2005 \backslash 0 \backslash \backslash \#$ B 3L

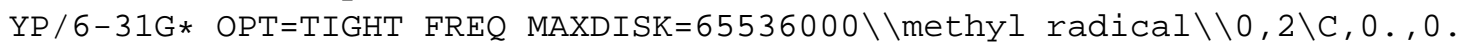
$, 0 . \backslash \mathrm{H}, 0 ., 0.1 .082753618 \backslash \mathrm{H}, 0.9376921392,0 \ldots,-0.541376809 \backslash \mathrm{H},-0.9376921392$ , $0 .,-0.541376809 \backslash \backslash$ Version=DEC-AXP-OSF / 1-G98RevA.11.3 $\backslash \mathrm{HF}=-39.8382922 \backslash \mathrm{S} 2$ $=0.753765 \backslash \mathrm{S} 2-1=0 . \backslash \mathrm{S} 2 \mathrm{~A}=0.750007 \backslash \mathrm{RMSD}=1.350 \mathrm{e}-09 \backslash \mathrm{RMSF}=4.499 \mathrm{e}-07 \backslash \mathrm{Dipole}=0$. , $0 ., 0 . \backslash \mathrm{PG}=\mathrm{D} 03 \mathrm{H}[\mathrm{O}(\mathrm{C} 1), 3 \mathrm{C} 2(\mathrm{H} 1)] \backslash \backslash \mathrm{Q}$ 


\section{$\mathrm{S}=\mathrm{CH}_{2}(\mathrm{~W} 1$ calculation $)$}

$1 \backslash 1 \backslash G I N C-A C 37 \backslash F r e q \backslash R B 3 L Y P \backslash 6-31 G(d) \backslash C 1 H 2 S 1 \backslash H W C 561 \backslash 10-$ Oct $-2005 \backslash 0 \backslash \backslash \#$ B 3LY $\mathrm{P} / 6-31 \mathrm{G} * \mathrm{OPT}=\mathrm{CALCALL}$ MAXDISK=13107200 \thiof $\backslash \backslash 0,1 \backslash \mathrm{S}, 0 ., 0 \ldots,-0.588151403$ $1 \backslash \mathrm{C}, 0.0 ., 1.0302599777 \backslash \mathrm{H}, 0 ., 0.9234507904,1.6144312916 \backslash \mathrm{H}, 0 .,-0.92345079$ $04,1.6144312916 \backslash \backslash$ Version=IA $64 \mathrm{~L}-\mathrm{G} 03 \mathrm{RevC} .02 \backslash$ State $=1-\mathrm{A} 1 \backslash \mathrm{HF}=-437.4623335 \backslash \mathrm{R}$ $\mathrm{MSD}=3.641 \mathrm{e}-09 \backslash \mathrm{RMSF}=3.313 \mathrm{e}-05 \backslash \mathrm{Dipole}=0,0 ., 0.7446479 \backslash \mathrm{DipoleDeriv=-0.128}$ $7113,0.0 .0 .,-0.1449245,0.0 .0 \ldots,-0.4193779,-0.1891044,0 \ldots, 0 \ldots, 0.0 .179$ $6809,0 \ldots, 0.0 \ldots, 0.5733279,0.1589078,0 \ldots, 0.0 \ldots,-0.0173782,-0.0723814,0 \ldots,-0$ $.0973752,-0.076975,0.1589078,0.0 ., 0 .,-0.0173782,0.0723814,0 ., 0.097375$ $2,-0.076975 \backslash$ Polar $=13.1154976,0 ., 18.9127605,0 ., 0 ., 39.5044017 \backslash \mathrm{PG}=\mathrm{C} 02 \mathrm{~V} \quad[\mathrm{C}$ $2(\mathrm{C} 1 \mathrm{~S} 1), \mathrm{SGV}(\mathrm{H} 2)] \backslash \mathrm{NImag}=0 \backslash \backslash 0.00802356,0 ., 0.02903238,0 \ldots, 0,0.43924629,-0$ $.03024608,0 ., 0.0 .11388818,0 .,-0.04940057,0 ., 0.0 .57391759,0 ., 0 .,-0.39$ $565598,0.0 .0 .63986627,0.01111126,0.0 .,-0.04182105,0.0 \ldots, 0.01535795$, $0 ., 0.01018409,0.00121180,0 \ldots,-0.26225851,-0.11586140,0 \ldots, 0.26959770,0 \ldots,-$ $0.01896906,-0.02179515,0 .,-0.12271638,-0.12210514,0 ., 0.12816752,0.1333$ $7536,0.01111126,0.0 \ldots,-0.04182105,0 \ldots, 0.0 .01535184,0 \ldots, 0.0 .01535795,0$. $, 0.01018409,-0.00121180,0 \ldots,-0.26225851,0.11586140,0 \ldots,-0.01752329,0.013$ $51792,0.0 .26959770,0 ., 0.01896906,-0.02179515,0 \ldots, 0.12271638,-0.1221051$ $4,0 .,-0.01351792,0.01052493,0 \ldots,-0.12816752,0.13337536 \backslash \backslash 0 ., 0 ., 0.0000009$ $3,0 ., 0 ., 0.00007682,0 ., 0.00004608,-0.00003888,0 .,-0.00004608,-0.0000388$ $8 \backslash \backslash \backslash 0$ 


\section{S2 PREDICI ${ }^{\circledR}$ model}

See ref. 19 for full description of the kinetic model used.

Intramolecular RAFT equilibrium

$$
\begin{array}{ll|r}
\text { Ainit }+ \text { Ainit-DB } & \leftrightarrow \text { A-RING } & k_{\text {close }} / k_{\text {open }} \\
\mathrm{P}(s)+\text { A-RING } & \rightarrow \text { AdeadIntra }(s) & k_{\text {tCross }}
\end{array}
$$

Decomposition and initiation

$$
\begin{array}{ll|l}
\mathrm{I} & \rightarrow \mathrm{I} \bullet+\mathrm{I} \bullet & k_{\mathrm{d}} \\
\mathrm{I} \bullet+\mathrm{M} & \rightarrow \mathrm{P}(1) & k_{\mathrm{i}}
\end{array}
$$

Pre-equilibrium

$$
\begin{array}{ll|r}
\mathrm{P}(s)+\text { Ainit-DB } & \rightarrow \text { QpreChain }(s) & k_{\beta \text { PreChainRight }} \\
\mathrm{A}(s)+\text { Ainit-DB } & \rightarrow \text { QpreArm }(s) & k_{\beta \text { PreArmRight }} \\
\text { QpreChain }(s) & \rightarrow \mathrm{P}(s)+\text { Ainit-DB } & k_{-\beta \text { PreChainLeft }} \\
\text { QpreChain }(s) & \rightarrow \mathrm{DB}-\mathrm{P}(s)+\text { Ainit } & k_{-\beta \text { PreChainRight }} \\
\text { QpreArm }(s) & \rightarrow \mathrm{A}(s)+\text { Ainit-DB } & k_{-\beta \text { PreArmLeft }} \\
\text { QpreArm }(s) & \rightarrow \mathrm{DB}-\mathrm{A}(s)+\text { Ainit } & k_{-\beta \text { PreArmRight }} \\
\mathrm{DB}-\mathrm{A}(s)+\text { Ainit } & \rightarrow \text { QpreArm }(s) & k_{\beta \text { PreArmLeft }}
\end{array}
$$

Arm growth

$$
\begin{array}{ll|l}
\mathrm{A}(s)+\mathrm{M} & \rightarrow \mathrm{A}(s+1) & k_{\mathrm{p}} \\
\mathrm{P}(s)+\mathrm{M} \rightarrow \mathrm{P}(s+1) & k_{\mathrm{p}} \\
\text { Ainit }+\mathrm{M} \rightarrow \mathrm{A}(1) & k_{\mathrm{ai}}
\end{array}
$$

Terminations

$$
\begin{array}{ll|r}
\mathrm{A}(s)+\mathrm{P}(r) & \rightarrow \text { Adead }(s+r) & k_{\mathrm{tArm}} \\
\mathrm{A}(s)+\mathrm{A}(r) & \rightarrow \mathrm{A}-\operatorname{coupled}(s+r) & k_{\mathrm{tArmArm}} \\
\mathrm{P}(s)+\mathrm{P}(r) & \rightarrow \mathrm{D}(s+r) & k_{\mathrm{t}}
\end{array}
$$

Star arm/chain RAFT equilibrium

$$
\begin{aligned}
& \mathrm{A}(s)+\mathrm{DB}-\mathrm{P}(r) \rightarrow \operatorname{Qstar1}(s)+\text { Qstar2 }(r) \mid k_{\beta S t a r} \\
& \mathrm{DB}-\mathrm{A}(s)+\mathrm{P}(r) \rightarrow \mathrm{Q} \operatorname{star} 1(s)+\mathrm{Q} \operatorname{star} 2(r) k_{\beta S t a r} \\
& \text { Qstar1 }(s) \quad \rightarrow \mathrm{A}(s) \quad k_{-\beta} \\
& \text { Qstar1 }(s) \quad \rightarrow \text { DB-A }(s) \quad k_{-\beta} \\
& \text { Qstar2 }(s) \quad \rightarrow \text { DB-P }(s) \quad k_{-\beta} \\
& \begin{array}{lll}
\operatorname{Qstar} 2(s) & \rightarrow \mathrm{P}(s) & k_{-\beta}
\end{array}
\end{aligned}
$$


star arm/arm RAFT equilibrium

$$
\begin{aligned}
& \mathrm{A}(s)+\mathrm{DB}-\mathrm{A}(r) \rightarrow \text { QstarStar1 }(s)+\text { QstarStar2 }(r) \mid k_{\beta S t a r} \\
& \text { QstarStar1 }(s) \rightarrow \mathrm{A}(s) \\
& \text { QstarStar1 }(s) \rightarrow \text { DB-A }(s) \\
& \text { QstarStar2 }(s) \rightarrow \mathrm{A}(s) \\
& \text { QstarStar2(s) } \rightarrow \text { DB-A }(s) \quad \frac{1}{2} k_{-\beta}
\end{aligned}
$$

Standard RAFT Equilibrium

$$
\begin{array}{ll|r}
\mathrm{P}(s)+\mathrm{DB}-\mathrm{P}(r) & \rightarrow \mathrm{Q} 1(s)+\mathrm{Q} 2(r) & k_{\beta} \\
\mathrm{Q} 1(s) & \rightarrow \mathrm{P}(s) & \frac{1}{2} k_{-\beta} \\
\mathrm{Q} 1(s) & \rightarrow \mathrm{DB}-\mathrm{P}(s) & \frac{1}{2} k_{-\beta} \\
\mathrm{Q} 2(s) & \rightarrow \mathrm{P}(s) & \frac{1}{2} k_{-\beta} \\
\mathrm{Q} 2(s) & \rightarrow \mathrm{DB}-\mathrm{P}(s) & \frac{1}{2} k_{-\beta}
\end{array}
$$

